PROCEEDINGS OF THE

AMERICAN MATHEMATICAL SOCIETY

Volume 135, Number 1, January 2007, Pages 217-227

S 0002-9939(06)08451-6

Article electronically published on June 29, 2006

\title{
MINIMIZING FUNCTIONS FOR AN UNCERTAINTY PRINCIPLE ON LOCALLY COMPACT GROUPS OF BOUNDED REPRESENTATION DIMENSION
}

\author{
EBERHARD KANIUTH
}

(Communicated by Michael T. Lacey)

\begin{abstract}
Let $G$ be a locally compact group of bounded representation dimension $d(G)$. Then, for any integrable function $f$ on $G$, the product of the measures of the support of $f$ and the support of its operator-valued Fourier transform on the dual space of $G$ is bounded below by $1 / d(G)$. We classify all functions for which equality holds and prove criteria for when such functions exist.
\end{abstract}

\section{INTRODUCTION}

Uncertainty principles on locally compact abelian groups $G$ have been studied during the past thirty years, and common to all of them is the assertion that a non-zero function $f$ in $L^{1}(G)$ or in $L^{2}(G)$ and its Fourier transform $\widehat{f}$ on the dual group $\widehat{G}$ cannot both be concentrated in some sense. Let Haar measures $m_{G}$ on $G$ and $\mu_{G}$ on $\widehat{G}$ be normalized such that the Plancherel formula holds, and for $f \in L^{1}(G)$ let

$$
A_{f}=\{x \in G: f(x) \neq 0\} \text { and } B_{f}=\{\chi \in \widehat{G}: \widehat{f}(\chi) \neq 0\} .
$$

Matolcsi and Szücs [1] have shown that $m_{G}\left(A_{f}\right) \mu_{G}\left(B_{f}\right) \geq 1$ for every $f \in L^{2}(G)$, $f \neq 0$. The minimizers, that is, functions for which equality holds, have been explicitly determined by Donoho and Stark [2] when $G$ is a finite cyclic group and for general finite abelian groups by Smith [14]. These minimizers are precisely the functions of the form $f(x)=c \varphi(a x)$, where $c \in \mathbb{C}, c \neq 0, a \in G$ and $\varphi$ is a character of some subgroup of $G$. If $G$ is a locally compact abelian group such that $G_{0}$, the connected component of the identity of $G$, is non-compact, then Hogan [6] has shown that for any non-zero $f \in L^{1}(G), m_{G}\left(A_{f}\right)=\infty$ or $\mu_{G}\left(B_{f}\right)=\infty$. Thus minimizers can only exist when $G_{0}$ is compact, and in this case it turned out that they are again exactly the functions given by the above formula with the only modification that now $\varphi$ is a character of some open compact subgroup of $G$ [10].

For an arbitrary locally compact group $G$, let $\widehat{G}$ denote the dual space of $G$, that is, the set of (equivalence classes) of irreducible unitary representations of $G$. If $m_{G}$ is a left Haar measure on $G$, the operator-valued Fourier transform of $f \in L^{1}(G)$

Received by the editors July 9, 2005 and, in revised form, August 1, 2005.

2000 Mathematics Subject Classification. Primary 43A30, 43A40.

(C)2006 American Mathematical Society Reverts to public domain 28 years from publication 
on $\widehat{G}$ is defined by

$$
\widehat{f}(\pi)=\pi(f)=\int_{G} f(x) \pi(x) d m_{G}(x)(\pi \in \widehat{G}) .
$$

In this general context, define $A_{f}$ and $B_{f}$ as above. Now assume that $G$ is of bounded representation dimension, that is,

$$
d(G)=\sup \{\operatorname{dim} \pi: \pi \in \widehat{G}\}<\infty .
$$

There exists a unique Borel measure $\mu_{G}$ on the topological space $\widehat{G}$ such that the Plancherel formula

$$
\int_{G}|f(x)|^{2} d m_{G}(x)=\int_{\widehat{G}} \operatorname{tr}\left(\pi(f) \pi(f)^{*}\right) d \mu_{G}(\pi)
$$

holds for all $f \in L^{1}(G) \cap L^{2}(G)[\underline{5}$.

It is easy to see that $m_{G}\left(A_{f}\right) \mu_{G}\left(B_{f}\right) \geq 1 / d(G)$ for any non-zero $f \in L^{1}(G) \cap$ $L^{2}(G)$ (Lemma 1.1). In 9 it has been claimed that equality can never occur if $G$ is a finite non-abelian group. However, this is not correct, as can be seen by considering the finite dihedral groups (Example 2.1). It was this observation that motivated our investigation. For a locally compact group of bounded representation dimension, we characterize the functions $f \in L^{1}(G) \cap L^{2}(G)$ for which $m_{G}\left(A_{f}\right) \mu_{G}\left(B_{f}\right)=1 / d(G)$ holds (Theorem 1.4). In contrast to the abelian case, due to the diversity of the groups in question, the existence of minimizing functions appears to be a difficult problem which we succeeded to solve only for some classes of groups of bounded representation dimension (Propositions 2.2, 2.4 and 2.5).

\section{Characterization OF Minimizing FunCtions}

Throughout the paper, $G$ will denote a locally compact group of bounded representation dimension. Note that these groups are exactly those which have an abelian normal subgroup of finite index 12. In particular, such groups are unimodular. For a unitary representation $\pi$ of $G$ on the Hilbert space $\mathcal{H}(\pi), d_{\pi}$ will denote the dimension of $\mathcal{H}(\pi)$ and $I_{\mathcal{H}(\pi)}$ the identity operator in $\mathcal{H}(\pi)$.

Lemma 1.1. Let $G$ be a locally compact group of bounded representation dimension. Then

$$
m_{G}\left(A_{f}\right) \mu_{G}\left(B_{f}\right) \geq 1 / d(G)
$$

for every non-zero function $f \in L^{1}(G) \cap L^{2}(G)$.

Proof. For $\pi \in \widehat{G}$, let $\left\{\xi_{\pi, j}: 1 \leq j \leq d_{\pi}\right\}$ be an orthonormal basis of $\mathcal{H}(\pi)$. Then

$$
\begin{aligned}
\operatorname{tr}\left(\pi(f)^{*} \pi(f)\right) & =\sum_{j=1}^{d_{\pi}} \int_{G} \int_{G} f(x) \overline{f(y)}\left\langle\pi(x) \xi_{\pi, j}, \pi(y) \xi_{\pi, j}\right\rangle d x d y \\
& \leq d_{\pi}\|f\|_{1}^{2}
\end{aligned}
$$


and hence, by the Plancherel formula and Hölder's inequality,

$$
\begin{gathered}
\|f\|_{2}^{2}=\int_{\widehat{G}} \operatorname{tr}\left(\pi(f) \pi(f)^{*}\right) d \mu_{G}(\pi) \\
\leq \mu_{G}\left(B_{f}\right) \cdot \sup _{\pi \in \widehat{G}}\left\{\operatorname{tr}\left(\pi(f) \pi(f)^{*}\right)\right\} \\
\leq \mu_{G}\left(B_{f}\right) d(G)\|f\|_{1}^{2} \\
\leq \mu_{G}\left(B_{f}\right) d(G)\left(\|f\|_{2} m_{G}\left(A_{f}\right)^{1 / 2}\right)^{2} \\
=d(G) m_{G}\left(A_{f}\right) \mu_{G}\left(B_{f}\right)\|f\|_{2}^{2},
\end{gathered}
$$

as required.

The main result of 7 implies that if $G$ is a group of bounded representation dimension and if the connected component $G_{0}$ of the identity is non-compact, then $m_{G}\left(A_{f}\right)=\infty$ or $\mu_{G}\left(B_{f}\right)=\infty$ for every non-zero function $f \in L^{1}(G) \cap L^{2}(G)$. Thus functions for which equality holds in Lemma 1.1 can only exist when $G_{0}$ is compact.

Before proceeding, we have to introduce some more notation. Let $N$ be a closed normal subgroup of $G$. We always consider $\widehat{G / N}$ as a closed subset of $\widehat{G}$ by identifying $\widehat{G / N}$ with the set of all $\pi \in \widehat{G}$ such that $\pi(x)=I_{\mathcal{H}(\pi)}$ for all $x \in N$. The term $G$-invariant character of $N$ means a continuous homomorphism from $N$ into the circle group $\mathbb{T}$ satisfying $\chi\left(y^{-1} x y\right)=\chi(x)$ for all $x \in N$ and $y \in G$. For such $\chi$, let

$$
\widehat{G}_{\chi}=\left\{\pi \in \widehat{G}: \pi(x)=\chi(x) I_{\mathcal{H}(\pi)} \text { for all } x \in N\right\} .
$$

Then $\widehat{G}_{\chi}$ is a non-empty closed subset of $\widehat{G}$. This can be seen as follows. For $\pi \in \widehat{G}$, let $P_{\pi}^{1}(G)$ denote the set of all normalized positive definite functions associated to $\pi$, that is, functions $\varphi$ of the form $\varphi(x)=\langle\pi(x) \xi, \xi\rangle, \xi \in \mathcal{H}(\pi),\|\xi\|=1$. Then $\left.\varphi\right|_{N}=\chi$ whenever $\pi \in \widehat{G}_{\chi}$. If $\rho$ is an element of the closure of $\widehat{G}_{\chi}$ in $\widehat{G}$, then every $\psi \in P_{\rho}^{1}(G)$ is a uniform on compacta limit of functions in $P_{\pi}^{1}(G), \pi \in \widehat{G}_{\chi}$ (see [1, Proposition 18.1.5]). It follows that $\left.\psi\right|_{N}=\chi$ for all $\psi \in P_{\rho}^{1}(G)$, and this shows that $\rho \in \widehat{G}_{\chi}$. Thus $\widehat{G}_{\chi}$ is closed in $\widehat{G}$. Clearly, $\widehat{G}_{\chi}$ is non-empty since every $\pi$ in the support of the induced representation $\operatorname{ind}_{N}^{G} \chi$ belongs to $\widehat{G}_{\chi}$. Moreover, if $N$ is compact, then $\widehat{G}_{\chi}$ is open in $\widehat{G}$ since $\widehat{N}$ is discrete.

For $d \in \mathbb{N}$, let $\widehat{G}_{d}=\left\{\pi \in \widehat{G}: d_{\pi}=d\right\}$. Then $\widehat{G}_{d(G)}$ is open in $\widehat{G}$ [1, Proposition 3.6.3].

Lemma 1.2. Let $N$ be a compact open normal subgroup of $G$ and let $\chi$ be a $G$ invariant character of $N$. Let $d \in \mathbb{N}$ and suppose that $d_{\pi}=d$ for $\mu_{G}$-almost all $\pi \in \widehat{G}_{\chi}$. Then

$$
m_{G}(N) \mu_{G}\left(\widehat{G}_{\chi}\right)=1 / d .
$$

In particular, if $d=d(G)$ and $f: G \rightarrow \mathbb{C}$ is defined by $f(x)=\overline{\chi(x)}$ for $x \in N$ and $f(x)=0$ for $x \notin N$, then

$$
m_{G}\left(A_{f}\right) \mu_{G}\left(B_{f}\right)=1 / d(G) .
$$

Proof. Normalize Haar measures so that $\left.m_{G}\right|_{N}=m_{N}$ and that the Plancherel formula holds. Since, by the orthogonality relations for compact groups, $\pi(f)=$ 
$\int_{N} \overline{\chi(x)} \pi(x) d m_{G}(x)=m_{N}(N) I_{\mathcal{H}(\pi)}$ for $\pi \in \widehat{G}_{\chi}$ and $\pi(f)=0$ for $\pi \in \widehat{G} \backslash \widehat{G}_{\chi}$, the hypothesis and the Plancherel formula yield

$$
\begin{aligned}
m_{G}(N) & =\int_{G}|f(x)|^{2} d m_{G}(x)=\int_{\widehat{G}} \operatorname{tr}\left(\pi(f)^{*} \pi(f)\right) d \mu_{G}(\pi) \\
& =m_{N}(N)^{2} \int_{\widehat{G}_{\chi}} d_{\pi} d \mu_{G}(\pi) \\
& =d \cdot m_{G}(N)^{2} \mu_{G}\left(\widehat{G}_{\chi}\right) .
\end{aligned}
$$

Since $B_{f}=\widehat{G}_{\chi}$, both statements of the lemma follow.

Lemma 1.3. Let $f \in L^{1}(G)$ be such that $\mu_{G}\left(B_{f}\right)<\infty$ and $d_{\pi}=d(G)$ for almost all $\pi \in B_{f}$. Then, for every $x \in G$, the function $\pi \rightarrow \operatorname{tr}\left(\pi(f) \pi\left(x^{-1}\right)\right)$ belongs to $L^{1}\left(\widehat{G}, \mu_{G}\right)$, and the function

$$
x \rightarrow \int_{\widehat{G}} \operatorname{tr}\left(\pi(f) \pi(x)^{-1}\right) d \mu_{G}(\pi)
$$

is continuous on $G$.

Proof. Let $\mathcal{H}$ be a $d(G)$-dimensional Hilbert space and realize all $\pi \in \widehat{G}_{d(G)}$ on $\mathcal{H}$. Let $\left\{\xi_{1}, \ldots, \xi_{d(G)}\right\}$ be an orthonormal basis of $\mathcal{H}$. Then, for any $\pi \in \widehat{G}_{d(G)}$ and $x \in G$,

$$
\operatorname{tr}\left(\pi(f) \pi\left(x^{-1}\right)\right)=\sum_{j=1}^{d(G)}\left\langle\pi\left(R_{x} f\right) \xi_{j}, \xi_{j}\right\rangle,
$$

where $R_{x} f(y)=f(y x)$. In particular, $\left|\operatorname{tr}\left(\pi(f) \pi\left(x^{-1}\right)\right)\right| \leq d(G)\|f\|_{1}$. Let $V=$ $B_{f} \cap \widehat{G}_{d(G)}$. Then, by hypothesis, $\mu_{G}(V)=\mu_{G}\left(B_{f}\right)<\infty$. Now, for every $x \in G$, the function $\pi \rightarrow \operatorname{tr}\left(\pi(f) \pi\left(x^{-1}\right)\right)$ is Borel measurable on $\widehat{G}$ (see [5, Satz 4]) and

$$
\begin{aligned}
\int_{\widehat{G}}\left|\operatorname{tr}\left(\pi(f) \pi\left(x^{-1}\right)\right)\right| d \mu_{G}(\pi) & =\int_{V}\left|\operatorname{tr}\left(\pi(f) \pi\left(x^{-1}\right)\right)\right| d \mu_{G}(\pi) \\
& \leq d(G)\|f\|_{1} \mu_{G}(V) .
\end{aligned}
$$

This proves the first statement. For any $x, y \in G$, we have

$$
\begin{aligned}
\left|\operatorname{tr}\left(\pi(f) \pi\left(x^{-1}\right)\right)-\operatorname{tr}\left(\pi(f) \pi\left(y^{-1}\right)\right)\right| & \leq \sum_{j=1}^{d(G)}\left|\left\langle\pi\left(R_{x} f-R_{y} f\right) \xi_{j}, \xi_{j}\right\rangle\right| \\
& \leq d(G)\left\|R_{x} f-R_{y} f\right\|_{1}
\end{aligned}
$$

and hence

$$
\begin{gathered}
\left|\int_{\widehat{G}} \operatorname{tr}\left(\pi(f) \pi\left(x^{-1}\right)\right) d \mu_{G}(\pi)-\int_{\widehat{G}} \operatorname{tr}\left(\pi(f) \pi\left(y^{-1}\right)\right) d \mu_{G}(\pi)\right| \\
\leq \int_{V}\left|\operatorname{tr}\left(\pi(f) \pi\left(x^{-1}\right)\right)-\operatorname{tr}\left(\pi(f) \pi\left(y^{-1}\right)\right)\right| d \mu_{G}(\pi) \leq d(G) \mu_{G}\left(B_{f}\right)\left\|R_{x} f-R_{y} f\right\|_{1} .
\end{gathered}
$$

Since the mapping $t \rightarrow R_{t} f$ from $G$ into $L^{1}(G)$ is continuous, the second statement of the lemma follows.

The following theorem provides an explicit description of functions $f \in L^{1}(G) \cap$ $L^{2}(G)$ satisfying $m_{G}\left(A_{f}\right) \mu_{G}\left(B_{f}\right)=1 / d(G)$ and shows that every such minimizing function is obtained by translation and scalar multiplication of functions $f$ as in 
Lemma 1.2. We would like to point out that even though the proof of 9 , Proposition 4.1] for finite groups is faulty, it considerably inspired the arguments of our proof.

Theorem 1.4. Let $G$ be a locally compact group of bounded representation dimension. Then a function $f \in L^{1}(G) \cap L^{2}(G)$ satisfies

$$
m_{G}\left(A_{f}\right) \mu_{G}\left(B_{f}\right)=1 / d(G)
$$

if and only if there exist a compact open normal subgroup $N$ of $G$ and a $G$-invariant character $\chi$ of $N$ such that $d_{\pi}=d(G)$ for almost all $\pi \in \widehat{G}_{\chi}$ and

$$
f(x)=\left\{\begin{array}{cl}
c \overline{\chi(a x)} & \text { for } \in a^{-1} N, \\
0 & \text { for } x \notin a^{-1} N
\end{array}\right.
$$

almost everywhere for some $a \in G$ and $c \in \mathbb{C}, c \neq 0$.

Proof. The sufficiency of the condition follows from Lemma 1.2. In fact, it is obvious that the class of minimizing functions is invariant under translation and scalar multiplication.

Conversely, let $f$ be a non-zero function in $L^{1}(G) \cap L^{2}(G)$ satisfying

$$
m_{G}\left(A_{f}\right) \mu_{G}\left(B_{f}\right)=1 / d(G) .
$$

Translating and normalizing $f$ if necessary, we may, and shall, assume that $\|f\|_{1}=$ 1 and $f(e) \neq 0$. By hypothesis, we have equality throughout in the chain of inequalities (1), (2) and (3) in the proof of Lemma 1.1. Equality in (1) and (2) implies that

(i) $\operatorname{tr}\left(\pi(f) \pi(f)^{*}\right)=d(G)$ for almost all $\pi \in B_{f}$.

Equality in (3) means that $\left\langle|f|, 1_{A_{f}}\right\rangle=\|f\|_{2}\left\|1_{A_{f}}\right\|_{2}$, whence

(ii) $|f(x)|=c \cdot 1_{A_{f}}(x)$ for some $c>0$ and almost all $x \in G$.

It follows from (i) and $\|f\|_{1}=1$ that $d_{\pi}=d(G)$ and that $\pi\left(f * f^{*}\right)$ is the identity operator $I_{\mathcal{H}(\pi)}$ in $\mathcal{H}(\pi)$, for almost all $\pi \in B_{f}$. We next recall that by Lemma 1.3, the function

$$
g: x \rightarrow \int_{\widehat{G}} \operatorname{tr}\left(\pi(f) \pi\left(x^{-1}\right)\right) d \mu_{G}(\pi)
$$

is continuous on $G$. Moreover, by the inversion formula [5, Satz 4], $g$ equals $f$ almost everywhere. Thus, replacing $f$ by $g$, we can assume that $f$ is continuous. In particular, $A_{f}$ is then open and (ii) holds for all $x \in G$.

For $\pi \in \widehat{G}$, let $\left\{\xi_{\pi, j}: 1 \leq j \leq d_{\pi}\right\}$ be an orthonormal basis of $\mathcal{H}(\pi)$, and with respect to that basis of $\mathcal{H}(\pi)$, let $\left(\pi(f)_{j k}\right)$ and $\left(\pi(x)_{j k}\right)$ be the matrix representation of $\pi(f)$ and $\pi(x)$, respectively. Thus

$$
\pi(x)_{j k}=\left\langle\pi(x) \xi_{\pi, j}, \xi_{\pi, k}\right\rangle, 1 \leq j, k \leq d_{\pi} .
$$

Since $\|f\|_{1}=1$ and $\left|\pi(t)_{j j}\right|=1$ for all $t \in A_{f}, 1 \leq j \leq d_{\pi}$, and $\pi \in B_{f}$, (i) implies that

$$
\begin{aligned}
\sum_{j=1}^{d_{\pi}}\left|\int_{G} \int_{G} f(x) \overline{f(y)} \pi\left(x y^{-1}\right)_{j j} d x d y\right| & =\sum_{j=1}^{d_{\pi}}\left\langle\pi(f) \xi_{\pi, j}, \pi(f) \xi_{\pi, j}\right\rangle \\
& =\operatorname{tr}\left(\pi(f) \pi(f)^{*}\right)=d(G) \\
& =d(G) \int_{G} \int_{G}|f(x)| \cdot|f(y)| d x d y
\end{aligned}
$$


for almost all $\pi \in B_{f}$. Hence, for each such $\pi \in B_{f}$, there exist constants $\lambda_{\pi, j}$, $1 \leq j \leq d_{\pi}$, of absolute value one such that, for all $x, y \in A_{f}$,

$$
\lambda_{\pi, j} f(x) \overline{f(y)} \pi\left(x y^{-1}\right)_{j j}=|f(x)| \cdot|f(y)| .
$$

Setting $y=x$ shows that $\lambda_{\pi, j}=1$ for all $j$. Since (ii) holds for all $x \in A_{f}$, choosing $y=e$ we obtain

$$
f(x) \pi(x)_{j j}=\frac{|f(x)| \cdot|f(e)|}{\overline{f(e)}}=f(e)
$$

for all $j$, all $x \in A_{f}$ and almost all $\pi \in B_{f}$. Now, for such $\pi$ and $x \in A_{f}$,

$$
\left|\left\langle\pi(x) \xi_{\pi, j}, \xi_{\pi, j}\right\rangle\right|=\left|\pi(x)_{j j}\right|=\left|\frac{f(e)}{f(x)}\right|=1
$$

for all $1 \leq j \leq d_{\pi}$. Since $\pi$ is unitary, it follows that $\pi(x)_{j k}=0$ for $j \neq k$. Thus, for almost all $\pi \in B_{f},\left(\pi(x)_{j k}\right)$ is the same scalar matrix, $\chi(x) E_{d_{\pi}}$ say, for all $x \in A_{f}$. We claim that this property characterizes the elements of $A_{f}$.

To that end, let $x \in G$ and $\lambda \in \mathbb{C}$ be such that $\pi(x)=\lambda I_{\mathcal{H}(\pi)}$ for almost all $\pi \in B_{f}$. Now

$$
\pi(f)_{j j}=\int_{A_{f}} f(t) \pi(t)_{j j} d t=f(e) m_{G}\left(A_{f}\right), 1 \leq j \leq d_{\pi},
$$

and $\pi(f)$ is a diagonal matrix since each $\pi(t)$ is a diagonal matrix. The inversion formula then yields

$$
\begin{aligned}
f(x) & =\int_{B_{f}}\left(\sum_{j=1}^{d_{\pi}}\left\langle\pi\left(x^{-1}\right) \xi_{\pi, j}, \pi\left(f^{*}\right) \xi_{\pi, j}\right\rangle\right) d \mu_{G}(\pi) \\
& =\int_{B_{f}}\left(\sum_{j=1}^{d_{\pi}} \pi\left(x^{-1}\right)_{j j} \overline{\pi(f)_{j j}}\right) d \mu_{G}(\pi) \\
& =\overline{f(e)} m_{G}\left(A_{f}\right) \int_{B_{f}} \operatorname{tr}\left(\pi\left(x^{-1}\right)\right) d \mu_{G}(\pi) \\
& =\overline{f(e)} m_{G}\left(A_{f}\right) \mu_{G}\left(B_{f}\right) d(G) \bar{\lambda} .
\end{aligned}
$$

It follows that if $x \in G$ is such that $\pi(x)$ is represented by the same scalar matrix for almost all $\pi \in B_{f}$, then $f(x) \neq 0$. This proves the above claim.

Since $e \in A_{f}$, an immediate consequence of this characterization of elements of $A_{f}$ is that $A_{f}$ is a subgroup of $G$, and of course $A_{f}$ is normal in $G$. Note that $A_{f}$ is open and compact since $f$ is continuous and $|f|$ is constant on $A_{f}$. Moreover, for $x \in A_{f}$, since $\pi(x)=\chi(x) I_{\mathcal{H}(\pi)}$ for almost all $\pi \in B_{f}, f(x)=\overline{f(e)} \overline{\chi(x)}$.

Clearly, the character $\chi$ is $G$-invariant, and the orthogonality relations for irreducible representations of the compact group $A_{f}$ show that

$$
B_{f}=\left\{\rho \in \widehat{G}: \rho(x)=\chi(x) I_{\mathcal{H}(\pi)} \text { for all } x \in A_{f}\right\}=\widehat{G}_{\chi} .
$$

Since $d_{\pi}=d(G)$ for almost all $\pi \in B_{f}$, the proof is finished.

Remark 1.5. (i) The reader will have observed that we always assumed the functions $f$ to be in $L^{1}(G) \cap L^{2}(G)$, whereas in the papers [6, 7, 9, 10, 11, 14, dealing with locally compact abelian groups, $f$ is allowed to be in $L^{1}(G)$ or in $L^{2}(G)$, respectively. In this context note that, for an arbitrary locally compact group $G$, if $f \in L^{2}(G)$ and $m_{G}\left(A_{f}\right)<\infty$, then $f \in L^{1}(G)$. On the other hand, if $G$ is abelian and 
$f \in L^{1}(G)$ satisfies $\mu_{G}\left(B_{f}\right)<\infty$, then it follows from duality theory and the Plancherel formula that $f \in L^{2}(G)$.

(ii) There exists a Plancherel measure $\mu_{G}$ on $\widehat{G}$ for every second countable unimodular group $G$ of type I (see [1, Theorem 18.8.2] or [4, Theorem 7.44], and for an explicit description for certain group extensions compare [8]). We have referred to [5] because the technical hypothesis that $G$ be second countable can be dropped when $G$ is a group with finite-dimensional irreducible representations. In addition, we shall use some very specific properties of $\mu_{G}$ for such groups in Section 2 .

\section{The EXISTENCE OF MINIMIZING FUnCTIONS}

In this section we are going to clarify the existence of minimizing functions for three different classes of groups of bounded representation dimension. However, we start with a simple example of a non-abelian finite group for which every $\pi \in \widehat{G}_{d(G)}$ belongs to $B_{f}$ for some minimizing function $f$.

Example 2.1. For $n \geq 2$, let $D_{n}$ denote the dihedral group of order $2^{n+1}$. Then $D_{n}$ is the group generated by two elements $a$ and $b$ subject to the relations $a^{2^{n}}=e$, $b^{2}=e$ and $b^{-1} a b=a^{-1}$. The dual space $\widehat{D_{n}}$ consists of 4 characters and $2^{n-1}-1$ irreducible representations of dimension 2. Let $Z=\left\{a^{2^{n-1}}, e\right\}$, the centre of $D_{n}$, and let $\chi$ be the non-trivial character of $Z$. The left regular representation $\lambda$ of $D_{n}$ decomposes into the direct sum of the two induced representations $\operatorname{ind}_{Z}^{D_{n}} 1_{Z}$ and $\operatorname{ind}_{Z}^{D_{n}} \chi$ of dimension $2^{n}$. Let $f$ denote the trivial extension of $\chi$ to $D_{n}$. Then $B_{f}$ consists of all irreducible subrepresentations of $\operatorname{ind}_{Z}^{D_{n}} \chi$, and each of these subrepresentations has dimension 2 and occurs with multiplicity 2 in $\operatorname{ind}_{Z}^{D_{n}} \chi$. This implies that $\left|B_{f}\right|=2^{n-2}$, and hence we obtain that

$$
m_{D_{n}}\left(A_{f}\right) \mu_{D_{n}}\left(B_{f}\right)=\frac{\left|A_{f}\right|}{2^{n+1}} \cdot 2\left|B_{f}\right|=\frac{1}{2}=\frac{1}{d\left(D_{n}\right)} .
$$

We now proceed to show that indeed every 2-dimensional irreducible representation of $D_{n}$ belongs to $B_{g}$ for some minimizing function $g$. The group $D_{n}$ is $n$-step nilpotent and, denoting by $Z_{k}\left(D_{n}\right), k=0,1, \ldots$, the ascending central series of $D_{n}, Z_{k}\left(D_{n}\right)$ is the cyclic group generated by $a^{2^{n-k}}, 0 \leq k \leq n-1$. Let $\chi_{k}$ be the non-trivial character of $Z_{k}\left(D_{n}\right) / Z_{k-1}\left(D_{n}\right)$ lifted to $Z_{k}\left(D_{n}\right)$ and let $f_{k}$ be the trivial extension of $\chi_{k}$ to all of $D_{n}, 1 \leq k \leq n-1$. Then $\left|A_{f_{k}}\right|=\left|Z_{k}\left(D_{n}\right)\right|=2^{k}$ and $D_{n} / Z_{k-1}\left(D_{n}\right)$ is isomorphic to $D_{n-k+1}$. Thus, substituting $n-k+1$ for $n$ in the first paragraph, we conclude that $\left|B_{f_{k}}\right|=2^{n-k-1}$. It follows that

$$
m_{D_{n}}\left(A_{f_{k}}\right) \mu_{D_{n}}\left(B_{f_{k}}\right)=\frac{\left|A_{f_{k}}\right|}{2^{n+1}} \cdot 2\left|B_{f_{k}}\right|=\frac{1}{2}=\frac{1}{d\left(D_{n}\right)} .
$$

Finally, observe that the union of all sets $B_{f_{k}}, 1 \leq k \leq n-1$, is precisely the set of all 2-dimensional irreducible representations of $D_{n}$.

Proposition 2.2. Let $G$ be a locally compact group such that its centre $Z(G)$ has finite index in $G$ and $G / Z(G)$ is abelian. Then minimizing functions exist if and only if $G_{0}$ is compact.

Proof. We only have to show that if $G_{0}$ is compact, then there exist minimizing functions. Since $Z(G)$ has finite index in $G$ and $G / Z(G)$ is abelian, the commutator subgroup of $G$ is finite [13, Theorem 4.12] and contained in $Z(G)$. Consequently, since $G_{0}$ is compact and $Z(G)$ is open, we find a compact open subgroup $N$ of $G$ 
such that $N \subseteq Z(G)$ and $G / N$ is abelian. Choose $\pi \in \widehat{G}$ such that $d_{\pi}=d(G)$. Then, since $N \subseteq Z(G)$, there exists a $G$-invariant character $\chi$ of $N$ such that $\pi \in \widehat{G}_{\chi}$. Now, since $G / N$ is abelian, every $\rho \in \widehat{G}_{\chi}$ is of the form $\rho=\pi \otimes \alpha$ for some $\alpha \in \widehat{G / N}$. It follows that $\widehat{G}_{\chi} \subseteq \widehat{G}_{d(G)}$, and then Lemma 1.2 shows that the trivial extension of $\bar{\chi}$ to all of $G$ is a minimizing function.

Let $G$ be a locally compact group and $N$ a closed normal subgroup of $G$. Then $G$ acts on $N$ through inner automorphisms and hence on $\widehat{N}$ by $(x, \tau) \rightarrow x \cdot \tau$, where $x \cdot \tau$ is defined by $x \cdot \tau(n)=\tau\left(x^{-1} n x\right), x \in G, n \in N$. For $\tau \in \widehat{N}$, let $S_{\tau}$ denote the stability group of $\tau$, that is, $S_{\tau}=\{x \in G: x \cdot \tau=\tau\}$.

Now, suppose that $G$ if of bounded representation dimension and hence has an abelian normal subgroup $A$ of finite index. Let $G_{F}$ denote the set of all elements $x \in G$ such that the set $\left\{y x y^{-1}: y \in G\right\}$, the conjugacy class of $x$, is relatively compact. Since $A \subseteq G_{F}, G_{F}$ is an open normal subgroup of finite index in $G$. The importance of $G_{F}$ in our context is due to the following facts. Let

$$
U=\left\{\tau \in \widehat{G_{F}}: S_{\tau}=G_{F}\right\} .
$$

Then $U$ is an open, $G$-invariant subset of $\widehat{G_{F}}$, and by Mackey's theory, for every $\tau \in U$, the induced representation $\operatorname{ind}_{G_{F}}^{G} \tau$ is irreducible (see [4, Theorem 6.39]). By Lemma 1 of [5], $U$ is dense in $\widehat{G_{F}}$ and $\widehat{G_{F}} \backslash U$ is a local $\mu_{G_{F}}$-zero set. Moreover, according to the construction of $\mu_{G}$ in $[5], \widehat{G} \backslash\left\{\operatorname{ind}_{G_{F}}^{G} \tau: \tau \in U\right\}$ is a local $\mu_{G}$-zero set in $\widehat{G}$.

Lemma 2.3. Let $G$ be a locally compact group with bounded representation dimension. Then $d(G)=\left[G: G_{F}\right] d\left(G_{F}\right)$.

Proof. Observe first that if $\pi \in \widehat{G}$, then $\pi \leq \operatorname{ind}_{G_{F}}^{G} \tau$ for some $\tau \in \widehat{G_{F}}$. This implies that $d(G) \leq\left[G: G_{F}\right] d\left(G_{F}\right)$. Conversely, let $U$ be as above and let $V=\left\{\tau \in \widehat{G_{F}}\right.$ : $\left.d_{\tau}=d\left(G_{F}\right)\right\}$. Then $U \cap V \neq \emptyset$ since $U$ is dense in $\widehat{G_{F}}$ and $V$ is non-empty and open. Now, for any $\tau \in U \cap V, \pi=\operatorname{ind}_{G_{F}}^{G} \tau$ is irreducible and

$$
d_{\pi}=\left[G: G_{F}\right] d_{\tau}=\left[G: G_{F}\right] \cdot d\left(G_{F}\right) .
$$

This shows that $d(G) \geq\left[G: G_{F}\right] d\left(G_{F}\right)$.

Proposition 2.4. Let $G$ be a group with bounded representation dimension such that $G_{F}$ is abelian. Then there exist minimizing functions for $G$ if and only if $G_{0}$ is compact, and in this case the indicator function of every compact open normal subgroup of $G$ is a minimizing function.

Proof. As pointed out in Section 1, compactness of $G_{0}$ is necessary. Conversely, let $G_{0}$ be compact and let $N$ be any compact open normal subgroup of $G$. Note that such subgroups $N$ exist since $G / G_{0}$ is totally disconnected and $G$ acts as a finite group of inner automorphisms on $G_{F}$. Again let $U$ be the set introduced before Lemma 2.3. Since $\widehat{G_{F} / N}$ is compact, $U \cap \widehat{G_{F} / N}$ is a co-null set in $\widehat{G_{F} / N}$. On the other hand, $d(G)=\left[G: G_{F}\right]$ (Lemma 2.3) and ind $G_{F}^{G} \tau \in \widehat{G}_{d(G)}$ for every $\tau \in U$. Now, Lemma 1.2 applies with $\chi$ the trivial character of $N$.

In the sequel, for a closed normal subgroup $H$ of $G,[H, G]$ will denote the closed subgroup of $G$ generated by all the commutators $h x h^{-1} x^{-1}, h \in H, x \in G$. 
Proposition 2.5. Let $G$ be a group of bounded representation dimension and let $H$ be the closed normal subgroup of $G$ defined by

$$
H=\left\{x \in G: \pi(x)=I_{\mathcal{H}(\pi)} \text { for all } \pi \in \widehat{G} \text { such that } d_{\pi}<d(G)\right\} .
$$

(i) Suppose that $H$ is non-compact. Then there exist minimizing functions if and only if $G_{0}$ is compact, and in this case the indicator function of every compact open normal subgroup of $G$ is a minimizing function.

(ii) Suppose that $H$ is compact. Then minimizing functions exist if there exists a compact open normal subgroup $N$ of $G$ such that $H \cap N \nsubseteq[N, G]$. More precisely, for any such $N$ there exists a $G$-invariant character $\chi$ of $N$ such that $\widehat{G}_{\chi} \subseteq \widehat{G}_{d(G)}$. Conversely, if minimizing functions exist and $\widehat{G / H}=\widehat{G} \backslash \widehat{G}_{d(G)}$ holds, then there exists a compact open normal subgroup $N$ of $G$ such that $H \cap N \nsubseteq[N, G]$.

Proof. (i) We only have to show that compactness of $G_{0}$ is sufficient. Choose any compact open normal subgroup $N$ of $G$. Then, by Lemma 1.2, the indicator function $1_{N}$ of $N$ is minimizing since $B_{1_{N}} \cap \widehat{G / H}$ is a set of measure zero because $H$ is non-compact.

(ii) Let $N$ be a compact open normal subgroup of $G$ such that $H \cap N \nsubseteq[N, G]$. For any $G$-invariant character $\chi$ of $N$, let $K_{\chi}=\{x \in N: \chi(x)=1\}$. Then, since the intersection of these $K_{\chi}$ equals $[N, G], H \cap N \nsubseteq K_{\chi}$ for some such $\chi$. Thus, if $\pi \in \widehat{G}_{\chi}$, then $\pi \notin \widehat{G / H}$ and hence $\widehat{G}_{\chi} \subseteq \widehat{G} \backslash \widehat{G / H} \subseteq \widehat{G}_{d(G)}$. By Lemma 1.2, the trivial extension to all of $G$ of $\bar{\chi}$ is minimizing.

Conversely, suppose that $\widehat{G / H}=\widehat{G} \backslash \widehat{G}_{d(G)}$, and let $N$ be a compact open normal subgroup of $G$ and $\chi$ a $G$-invariant character of $N$ such that $\mu_{G}\left(\widehat{G}_{\chi}\right)=$ $\mu_{G}\left(\widehat{G}_{\chi} \cap \widehat{G}_{d(G)}\right)$ (Theorem 1.4). Then $\widehat{G}_{\chi} \cap \widehat{G / H}=\emptyset$ since otherwise this set is nonempty and open in $\widehat{G}$ and hence has positive measure. Finally, $\widehat{G}_{\chi} \cap \widehat{G / H}=\emptyset$ implies that $H \cap N \nsubseteq K_{\chi}$, whence $H \cap N \nsubseteq[N, G]$. Indeed, suppose that $H \cap N \subseteq K_{\chi}$. Then we can define a function $\eta$ on $H N$ by $\eta(h x)=\chi(x)$ for $h \in H, x \in N$. It is easy to verify that $\eta$ is a $G$-invariant character of $H N$, and hence there exists $\pi \in \widehat{G}$ such that $\pi(y)=\eta(y) I_{\mathcal{H}(\pi)}$ for all $y \in H N$. Then $\pi \in \widehat{G}_{\chi} \cap \widehat{G / H}$, a contradiction.

Corollary 2.6. Suppose that the dimension of every irreducible representation of $G$ equals either 1 or $d(G)$.

(i) If $G \neq G_{F}$, then $G_{F}$ is abelian and hence Proposition 2.4 applies.

(ii) If $G=G_{F}$, then minimizing functions exist if and only if there exists a compact open normal subgroup $N$ of $G$ such that $[G, G] \cap N \nsubseteq[N, G]$.

Proof. For (i), we only have to observe that since $d(G)=\left[G: G_{F}\right] d\left(G_{F}\right)$, the hypothesis implies that $d\left(G_{F}\right)=1$, whence $G_{F}$ is abelian.

If $G=G_{F}$, then $[G, G]$ is compact. Indeed, since $G$ is a group of bounded representation dimension and relatively compact conjugacy classes, the dual space $\widehat{G}$ is a Hausdorff space 3 and hence $\widehat{G /[G, G]}$, the set of all characters of $G$, is open in $\widehat{G}$. This implies that $[G, G]$ is compact. Since $\left.\widehat{G}_{d(G)}=\widehat{G} \backslash \widehat{G /[G, G}\right]$ by hypothesis, (ii) follows from Proposition 2.5(ii).

We close the paper with two lemmas which might be useful when considering specific examples. 
Lemma 2.7. Let $K$ be a compact normal subgroup of $G$ such that $d(G / K)=$ $d(G)$, and suppose that there exist minimizing functions for $G / K$. Then there exist minimizing functions for $G$.

Proof. Let Haar measures on $G, K$ and $G / K$ be normalized so that Weil's formula holds and $m_{K}(K)=1$. Then $\mu_{G / K}$ equals the measure induced from $\mu_{G}$ on $\widehat{G / K} \subseteq$ $\widehat{G}$. Now, let $g \in L^{1}(G / K)$ satisfy

$$
m_{G / K}\left(A_{g}\right) \mu_{G / K}\left(B_{g}\right)=1 / d(G / K)=1 / d(G),
$$

and define $f \in L^{1}(G)$ by $f(x)=g(x K), x \in G$. Then $m_{G}\left(A_{f}\right)=m_{G / K}\left(A_{g}\right)$, and the orthogonality relations for irreducible representations of $K$ show that $B_{f} \subseteq$ $\widehat{G / K}$ and hence $B_{f}=B_{g}$. It follows that $m_{G}\left(A_{f}\right) \mu_{G}\left(B_{f}\right)=1 / d(G)$.

Lemma 2.8. Let $G$ be a projective limit of groups $G_{\alpha}=G / K_{\alpha}$. Then the following two conditions are equivalent:

(i) There exist minimizing functions for $G$.

(ii) For some $\alpha_{0}$, there exist minimizing functions for $G_{\alpha}$ for all $\alpha \geq \alpha_{0}$.

Proof. We first show (ii) $\Rightarrow$ (i). Since $\widehat{G}=\bigcup_{\alpha} \widehat{G_{\alpha}}\left[12\right.$, Lemma 2], there exists $\alpha_{1}$ such that $d\left(G_{\alpha}\right)=d(G)$ for all $\alpha \geq \alpha_{1}$. Fix any $\alpha$ such that $\alpha \geq \alpha_{1}$ and $\alpha \geq \alpha_{0}$. Since there exist minimizing functions for $G / K_{\alpha}$, (i) follows from Lemma 2.7.

Now, assume (i) and let $N$ be a compact open normal subgroup of $G$ and $\chi$ a $G$-invariant character of $N$ such that $d_{\pi}=d(G)$ for almost all $\pi \in \widehat{G}_{\chi}$ (Theorem 1.4). Since $N$ is open, $K_{\alpha} \subseteq N$ for all $\alpha \geq \alpha_{1}$, and $N$ is the projective limit of the groups $N / K_{\alpha}, \alpha \geq \alpha_{1}$. Therefore, since $\widehat{N}=\bigcup_{\alpha \geq \alpha_{1}} \widehat{N / K_{\alpha}}$, there exists $\alpha_{0} \geq \alpha_{1}$ such that $K_{\alpha} \subseteq N$ and $\chi \in \widehat{N / K_{\alpha}}$ for all $\alpha \geq \alpha_{0}$. Fix such an $\alpha$ and set $K=K_{\alpha}$ and $H=G / K_{\alpha}$, and define an $H$-invariant character $\eta$ on $N / K$ by $\eta(x K)=\chi(x), x \in N$. Then $\widehat{H}_{\eta}=\widehat{G}_{\chi}$ and, normalizing Haar measures as in the proof of Lemma 2.7, we have $\mu_{H}=\left.\mu_{G}\right|_{\widehat{H}}$. It follows that $d_{\rho}=d(G)=d(H)$ for $\mu_{H}$-almost all $\rho \in \widehat{H}_{\eta}$, as required.

\section{ACKNOWLEDGEMENT}

The author is indebted to the referee for his careful reading of the manuscript and his comments that led to several clarifications.

This work was completed during a stay at the Erwin Schrödinger Institute in Vienna in April 2005. The author thanks the organizers of the special semester on "Modern Methods of Time-Frequency Analysis" and the ESI for the invitation and hospitality.

\section{REFERENCES}

[1] J. Dixmier, $C^{*}$-algebras, North-Holland, Amsterdam, 1977. MR0458185 (56:16388)

[2] D.L. Donoho and P.B. Stark, Uncertainty principles and signal discovery, SIAM J. Appl. Math. 49 (1989), 906-931. MR0997928 (90c:42003)

[3] S. Echterhoff and E. Kaniuth, Certain group extensions and twisted covariance algebras with generalized continuous trace, Lecture Notes in Math. 1359 (1988), 159-169. MR0974312 (90c:46083)

[4] G.B. Folland, A course in abstract harmonic analysis, CRC Press, Boca Raton, 1995. MR1397028 (98c:43001) 
[5] W. Hauenschild and E. Kaniuth, Harmonische Analyse auf Gruppen mit endlichdimensionalen irreduziblen Darstellungen, Math. Z. 144 (1975), 239-256. MR0390125 $(52: 10951)$

[6] J.A. Hogan, A qualitative uncertainty principle for locally compact abelian groups, Miniconferences on harmonic analysis and operator algebras (Canberra 1987), Proc. Centre Math. Anal. Austral. Nat. Univ., Vol. 16 (1988), pp. 133-142. MR0953989 (89k:43006)

[7] J.A. Hogan, A qualitative uncertainty principle for unimodular groups of type I, Trans. Amer. Math. Soc. 340 (1993), 587-594. MR.1102222 (94b:43003)

[8] A. Kleppner and R.L. Lipsman, The Plancherel formula for group extensions, Ann. Sci. Ec. Norm. Sup. 5 (1972), 459-516. MR0342641 (49:7387)

[9] G. Kutyniok, A weak qualitative uncertainty principle for compact groups, Illinois J. Math. 47 (2003), 709-724. MR2007232 (2004h:43006)

[10] G. Kutyniok, A qualitative uncertainty principle for functions generating a Gabor frame on LCA groups, J. Math. Anal. Appl. 279 (2003), 580-596. MR.1974047(2004d:43006)

[11] T. Matolcsi and J. Szücs, Intersection des mesures spectrales conjugèes, C.R. Acad. Sci. Paris 277 (1973), 841-843. MR0326460 (48:4804)

[12] C.C. Moore, Groups with finite dimensional irreducible representations, Trans. Amer. Math. Soc. 166 (1972), 401-410. MR0302817 (46:1960)

[13] D.J.S. Robinson, Finiteness conditions and solvable groups. I, Springer, Berlin-HeidelbergNew York, 1972.

[14] K.T. Smith, The uncertainty principle on groups, SIAM J. Appl. Math. 50 (1990), 876-882. MR.1050918 (91i:94008)

Institut für Mathematik, Universität Paderborn, D-33095 Paderborn, Germany

E-mail address: kaniuth@math.uni-paderborn.de 